\title{
GOES-16 and GOES-17 ABI INR Assessment
}

\author{
Bin $\operatorname{Tan}^{1,2}$, John Dellomo ${ }^{1,3}$, Robert Wolfe $^{1}$, and Alan Reth ${ }^{1,4}$ \\ 1. Goddard Space Flight Center, Greenbelt, MD \\ 2. Science Systems and Applications, Inc., Lanham, MD \\ 3. Global Science \& Technology, Inc., Greenbelt, MD \\ 4. Chesapeake Aerospace, LLC, Annapolis, MD
}

\begin{abstract}
The first two satellites of the US Geostationary Operational Environmental Satellite R-Series (GOES-R) were launched on November 19, 2016 and March 1, 2018 respectively. GOES-16 officially became GOES East on December 18, 2017, and the designation of GOES-17 as GOES West occurred on February 12 2019. The Advanced Baseline Imager (ABI) is the primary instrument on GOES-16 and GOES-17 for imaging Earth's surface and atmosphere to significantly improve the detection and observation of severe environmental phenomena. The Image Navigation and Registration (INR) Performance Assessment Tool Set (IPATS) was developed to assess INR performance of GOES-R series ABI images. In this paper, we first describe the assessment of IPATS algorithm accuracy. Next, we present the relationship between view zenith angle (VZA) and the quality of the IPATS measurements. Lastly, we present GOES-16 and GOES-17 navigation (NAV) assessments results from flight data spanning from the start of INR assessment to June 2019. The results show a) IPATS "stair step" measurement error is less or equal to 0.06 ABI pixel with IPATS baseline configuration; b) VZA is an effective filter to exclude outliers of the measurements; and c) ABI INR for both satellites has improved over time as postlaunch tests (PLT) were performed and corrections applied. This paper also shows that the post-launch INR tuning of GOES-17 was much shorter than GOES-16.
\end{abstract}

Keywords: GOES-16, GOES-17, INR, ABI, Remote Sensing, Satellite

\section{INTRODUCTION}

The US Geostationary Operational Environmental Satellite R-series (GOES-R) satellites were launched on November 19 2016 and March 1 2018. They were designated GOES-16 and GOES-17 respectively upon reaching geostationary orbit. After checkout and calibration, GOES-16 was relocated to its operational location of 75.2 degrees west and officially became GOES East on December 18, 2017. GOES-17 was relocated to 137.2 degrees west on November 13, 2018 and officially became GOES West on February 12 2019. The Advanced Baseline Imager (ABI) is the primary instrument on the GOES satellites for imaging Earth's surface and atmosphere for weather forecast, detection and observation of severe environmental phenomena, and climate change studies [1]. The GOES-R navigation system was designed to support the very accurate image navigation and registration (INR) that many of these applications require [2].

There are three types of ABI images: Full Disk (FD) and Continental United States (CONUS) and Mesoscale (MESO) [3]. The ABI FD and CONUS images are composite of multiple scans. The scans of ABI data are gridded into the ABI fixed grid coordinate system by geometric correction of each scan. The fixed grid coordinate system is a two-dimensional angle space centered at the idealized location of a satellite in geosynchronous orbit [4]. The geolocation accuracy of ABI data is evaluated through INR assessments, an important part of the post-launch calibration and validation (Cal/Val) activities.

The INR Performance Assessment Tool Set (IPATS) was developed under the auspices of the NASA's GOES-R Flight Project for independent verification of ABI and Geostationary Lightning Mapper (GLM) INR for both GOES-16 and GOES-17 [5] [6]. It was built to measure four INR accuracy metrics: Navigation (NAV), Band-to-band Registration (BBR), Frame-to-Frame Registration (FFR) and Scene-to-Scene Registration (SSR). IPATS has been monitoring the INR accuracy of ABI images continuously starting when the GOES satellites were activated in their checkout location, 89.5 degrees west. Note that this paper focuses on the ABI NAV results. 
In this paper, we first describe the assessment of the IPATS NAV algorithm's accuracy. Next, we present the relationship between view zenith angle (VZA) and the quality of the NAV measurements. Lastly, we present the latest GOES-16 and GOES-17 NAV results from flight data spanning from the start of Post Launch Testing (PLT) through June 2019.

\section{IPATS NAV Algorithm Accuracy Assessment}

The INR error is composed of two components: INR intrinsic error and the measurement error:

$$
\epsilon_{I N R}=\epsilon_{I N R-i n t r i n s i c}+\epsilon_{M E}
$$

where $\epsilon_{I N R}$ is the IPATS measured INR error, $\epsilon_{I N R-i n t r i n s i c}$ is the INR-intrinsic error of the ABI system, and $\epsilon_{M E}$ is the measurement error due to uncertainty in the IPATS algorithms.

(a)

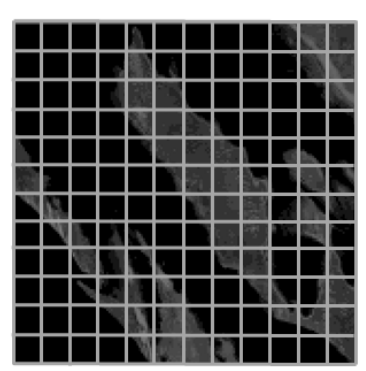

(c)

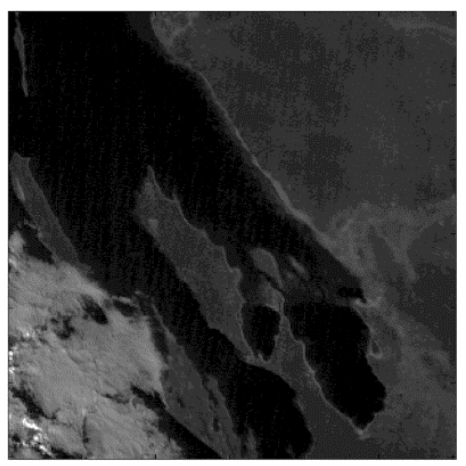

(b)

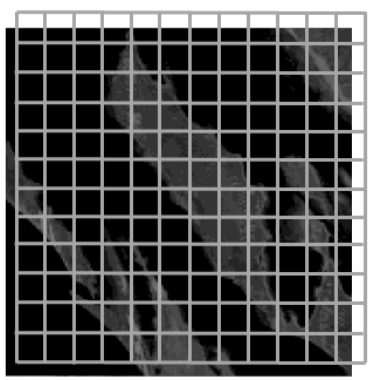

(d)

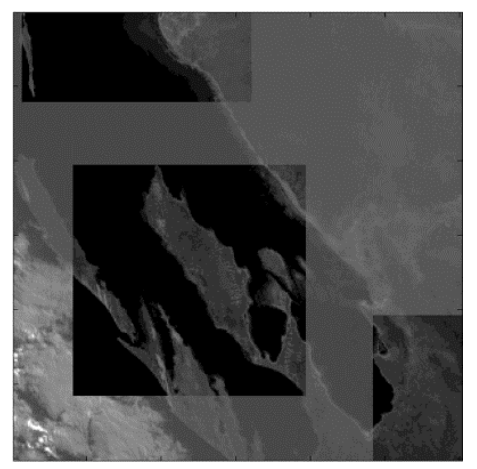

Figure 1. (a) Landsat chips are aggregated to GOES resolution without an induced shift. (b) Landsat chips are aggregated to GOES resolution with a half pixel induced shift in both the east-west and north-south directions. (c) Original GOES resolution image. (d) GOES resolution image with embedded aggregated chips (darker squares). Each grid cell in (a) and (b) represents a GOES resolution pixel. Note that the grid shows the concept of generating known shift GOES resolution data. The actual grid cells are much smaller than them in (a) and (b). The Landsat pixels falling into a grid cell are aggregated to one pixel in GOES resolution.

The image-matching algorithm, used in the estimation of all INR performance metrics, is the core of IPATS. In NAV assessment, IPATS measures the location of ABI subsets with respect to Landsat subsets, which are called Landsat chips in this paper. In BBR, IPATS compares ABI subsets from different spectral channels acquired at the same time, and in FFR assessments, IPATS compares ABI subsets from the same spectral channel acquired at different times. Stair-step error is one measurement error introduced by the image-matching algorithm. The name "stair-step error" comes from the fact measured INR error plotted against the true INR error resembles a stair-step shape. The difference between measured and true error is therefore oscillatory, and the frequency and the amplitude of the oscillation depend on the spatial resolution at which the image correlation is performed. The frequency is higher and the amplitude is smaller when matching images 
at higher resolution [7]. In the case of ABI NAV, the Landsat chips are formed by transforming Landsat images to the viewpoint of the GOES satellite at a particular orbital longitude. The Landsat chips, which are considered as truth data, have much finer resolution than the GOES imagery. To minimize the measurement error due to this stair-step error, the image-matching process is performed at an intermediate target resolution. The ABI data is up-sampled to this target resolution, and the truth data is down-sampled so that the two images are at a common resolution for correlation. We introduce the term 'sub-pixel factor' (SPF) to the degree of up-sampling applied to ABI images. For instance, a SPF of 2 means that the $\mathrm{ABI}$ images are up-sampled to half an $\mathrm{ABI}$ pixel before matching occurs.

(a)

RMSE of Measured EW Misregistration
\[ (\mathrm{SPF}=1) \]

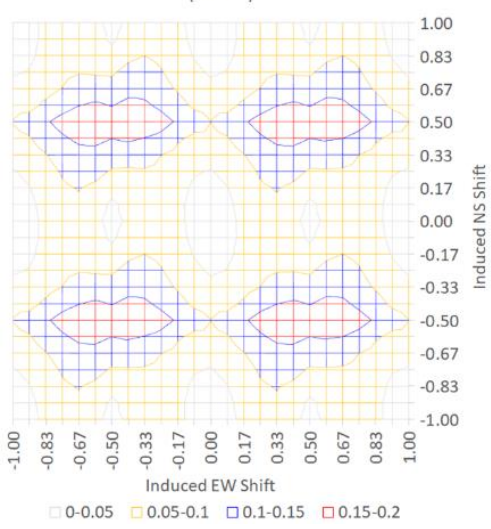

(c)

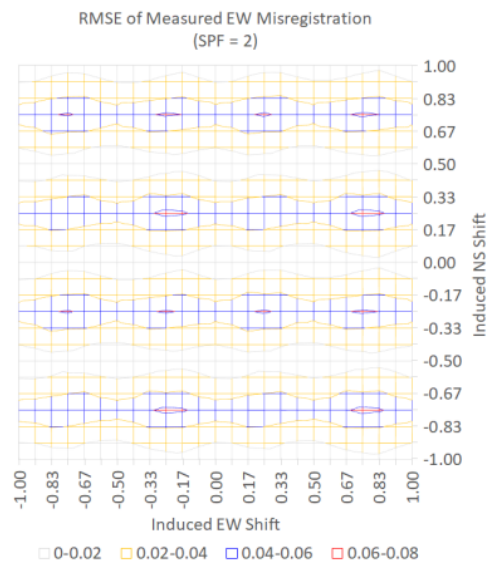

(b)

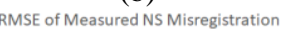
$(\mathrm{SPF}=1)$

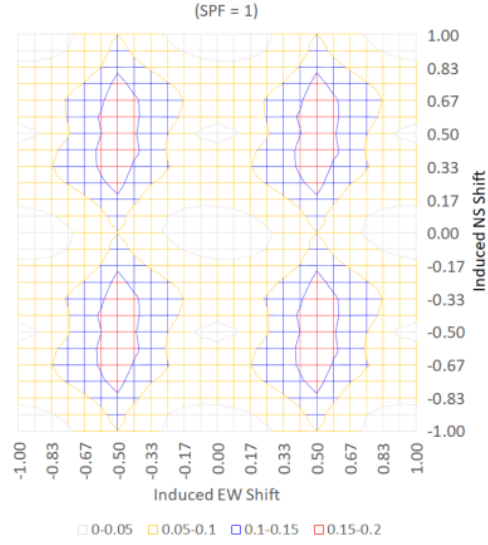

(d)

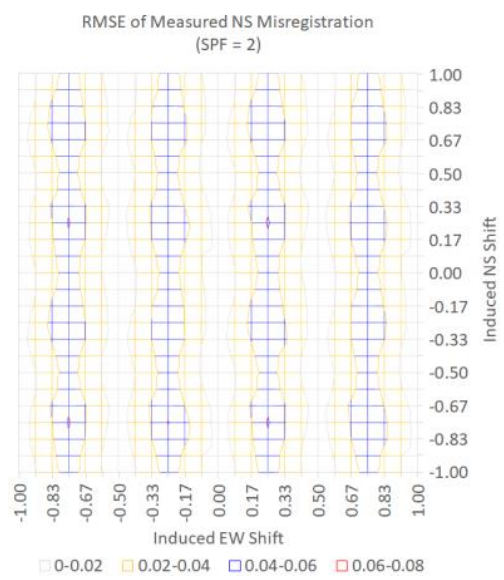

Figure 2. The RMSE (unit: pixel size) of NAV errors measured by 136 chips against induced shifts (unit: pixel size) which are intrinsic errors. Panel (a) and (b) are the results when SPF equals to 1. Panel (c) and (d) are the results when SPF equals to 2.

To estimate the stair-step component of $\epsilon_{M E}$ and determine the optimal target image resolution, we ran IPATS to process test images with known, intentionally-induced INR errors. Figure 1 shows how GOES resolution images were generated with induced shifts. A grid is overlaid on top of the Landsat chip. The size of the grid cell is the same as the spatial resolution of GOES. The Landsat pixels falling into a grid cell are aggregated to one GOES resolution pixel. The sub-pixel shifts are induced into aggregated GOES resolution images when the grid is shifted as shown in panel (b). The induced shift is the intrinsic shift in the GOES resolution images, $\boldsymbol{\epsilon}_{I N R \text {-intrinsic }}$. The shifted and aggregated Landsat chips are embedded into GOES resolution images and then the GOES resolution images are assessed by IPATS to measure the shift, which is $\boldsymbol{\epsilon}_{I N R}$. Now $\boldsymbol{\epsilon}_{M E}$ can be evaluated with known $\boldsymbol{\epsilon}_{I N R}$ and $\boldsymbol{\epsilon}_{I N R \text {-intrinsic }}$. In this test, 136 Landsat chips were aggregated and embedded in the GOES resolution image. 
Table 1. The RMSE of measured errors when there is no induced shifts. The unit of RMSE is pixel size.

\begin{tabular}{ccc} 
SPF & RMSE (EW) & RMSE (NS) \\
\hline 1 & 0.005 & 0.004 \\
\hline 2 & 0.010 & 0.012 \\
\hline 3 & 0.013 & 0.014 \\
\hline 4 & 0.013 & 0.015 \\
\hline 6 & 0.017 & 0.018 \\
\hline 12 & 0.017 & 0.018
\end{tabular}

Figure 2 shows the RMSE (root mean square error) of the 136 measured errors against induced errors in east-west (EW) and north-south (NS) directions when the SPF equals to 1 or 2. RMSE values are considered one of the main components of the measurement error $\boldsymbol{\epsilon}_{M E}$. For SPF equals to 1, peak RMSE is about 0.19 pixels and the error has a frequency of one pixel. Peak RMSE is about 0.06 pixels and the frequency is half pixel when SPF equals to 2 . The intrinsic error in one direction leads to measurement error in both directions. For example, there are measurement error in both EW and NS directions when intrinsic error at NS direction is 0 . It is observed that RMSE is close to but not exactly 0 when the intrinsic error is 0 in both directions (Table 1) because of other error sources, e.g. imperfect resample algorithm, the possible true minor mismatch between Landsat chips and ABI subsets, and the insufficient spatial/spectral information in the coarse resolution image. The RMSE for the null case increases slightly as SPF increases because of more errors introduced when interpolating the $\mathrm{ABI}$ images to finer resolution.

(a)

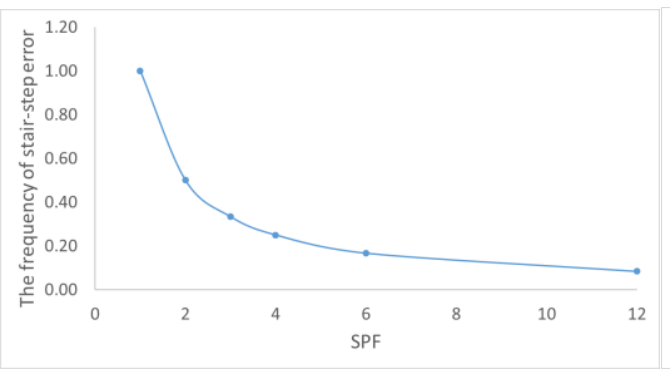

(b)

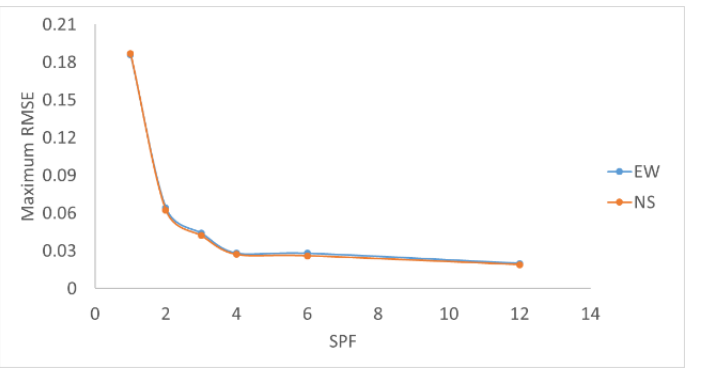

Figure 3. (a) The frequency of stair-step error against SPF values. The frequency is same in both EW and NS directions. (b) Maximum RMSE against SPF values in both EW and NS directions.

The frequency of the stair-step error, equal to the size of the target resolution of the image-matching, and the amplitude of RMSE depends on the SPF value (Figure 3) [7]. For cases with non-zero induced shifts, the maximum RMSE decreases with increasing SPF. At the GOES resolution (SPF =1), the maximum RMSE is about 0.19 pixels. There is a significant drop, from 0.19 to 0.06 pixels, from SPF of 1 to 2. It drops slowly from 0.06 to 0.02 pixels when SPF increases from 2 to 12. The measurement quality improved slightly but the computation time increases significantly because the computation time of the image-matching algorithm has O (SPF2) time complexity. After examining the tradeoff between the accuracy and the computation cost, the SPF was set to 2 in IPATS baseline configuration. The measurement error is only $1 \%$ of the pixel size when the intrinsic error is close to 0 (Table 1). This is sufficient for assessing the NAV accuracy.

\section{The impact of View Zenith Angle on NAV assessment}

IPATS applies post-processing filters on measured NAV errors to reject poor quality measurements and obtain a better estimate of the intrinsic NAV errors. The sun zenith angle (SZA) and measurement uncertainties (MU) filter remove most of the poor-quality NAV measurements. Statistics based filters, Mean absolute deviation (MAD), removes extreme outlier measurements and then Short Term AbNormal Detection (STAND) restores the abnormal large readings associated with significant intrinsic errors [6]. 
VZA filter was originally not included in IPATS post-processing because there are only 28 out of 644 chip locations with high VZA (more than 75 degrees) from the view of GOES-16 (Figure 4a and c). Nevertheless, VZA is a critical character of the satellite images. There has been very extensive research on the relationship between VZA and quality of the radiance observed by the satellite sensors and biogeophyical parameters retrieved from the satellite images [8][9][10]. However, there are few studies on the impact of VZA on the registration of the satellite images with reference data. The effect of differences in VZA is twofold. First, the atmosphere refracts the rays of light causing the apparent position of the viewpoint on the ground to shift toward the zenith. Second, the pixel footprint increases with increasing VZA. There is a higher possibility that a pixel with larger footprint is contaminated by clouds. Also, coarser resolution images contain less ground spatial information.

(a)

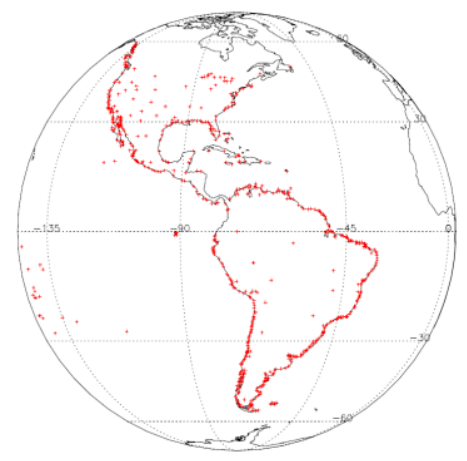

(c)

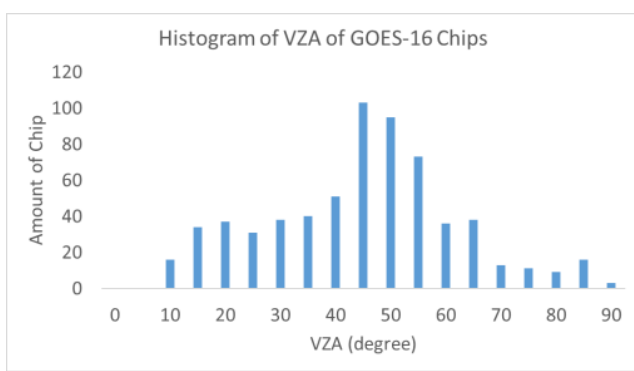

(b)

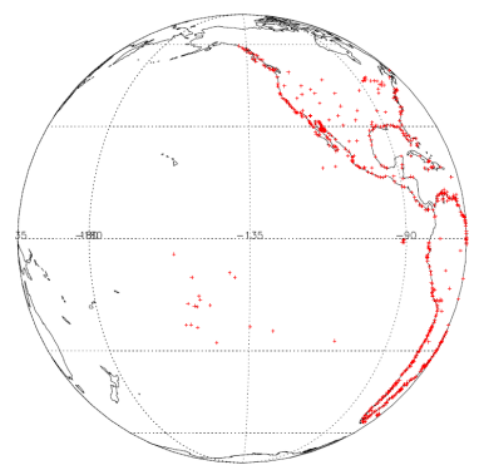

(d)

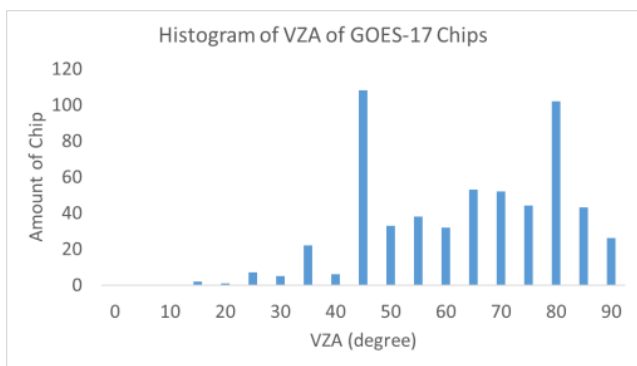

Figure 4. The chip locations and VZA of chip locations in the view of GOES-16 (a) (c) and GOES-17 (b) (d). There are 644 chip locations in the view of GOES-16 and 574 chip locations in the view of GOES-17.

Due to the nature of the satellite location, VZA of most of the Landsat chip locations for GOES-16 is small or moderate (Figure 4a and 4c). On the other hand, VZA of GOES-17 Landsat chip location is more significant, especially for the chips in the South America. There are 153 out of 574 chip locations with large VZA (more than 75 degrees). The GOES-17 chips with large VZA have very high rate of abnormal significant measured error reported.

Figure 5 shows the relationship between the measured NAV error and VZA in two FD channel 2 scenes on November 30 2018. The variation of the measurements increases significantly from less than $5 \mu \mathrm{rad}$ to around $100 \mu \mathrm{rad}$ in both EW and NS directions when VZA is more than 75 degrees. The MAD filter rejected 27 of 180 NAV chip measurements of the scene at 15:30 UTC and 23 of 44 measurements of the scene at 14:10 UTC. In the scene at 15:30 UTC, the 26 of 27 rejected measurements are with VZA larger than 75 degrees and there are only 11 of 153 accepted measurements are with VZA larger than 75 degrees. In the scene at 14:10 UTC, the VZA of 22 out of the 23 rejected NAV chip measurements are larger than 75 degrees. There are 8 of 21 accepted measurements whose VZA are larger than 75 degrees.

The MAD filter successfully rejected unrealistic significant measured errors (Figure 5). However, there are two issues if using MAD filter to clean up the NAV chip measurements with high VZA. First, the accepted measurements with high VZA could be in a reasonable range coincidentally but not represent intrinsic INR error. Second, the STAND filter uses 
MAD acceptance and rejection rates to detect true anomalous performance. There were 23 of 44 measurements in 14:10 UTC scene rejected by MAD filter and therefore it was marked as having large intrinsic errors by the STAND filter. A detail examination of the INR records and the comparison between FD scenes at 14:10 UTC and 15:30 UTC shows that there is no large intrinsic errors with the scene at 14:10 UTC. The high ratio of poor quality measurements is because only earth limb was lit up and most of the measurements were with high VZA (Figure 6).

(a)

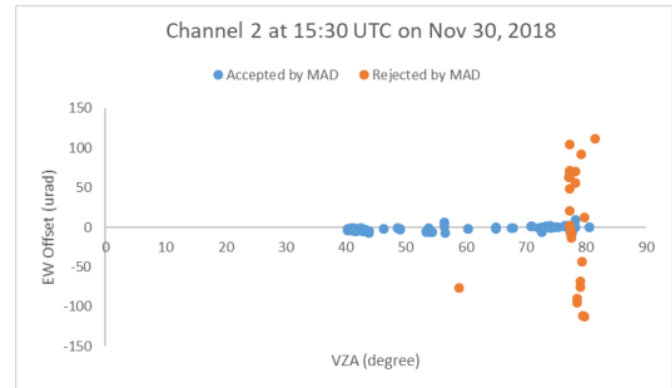

(c) (b)

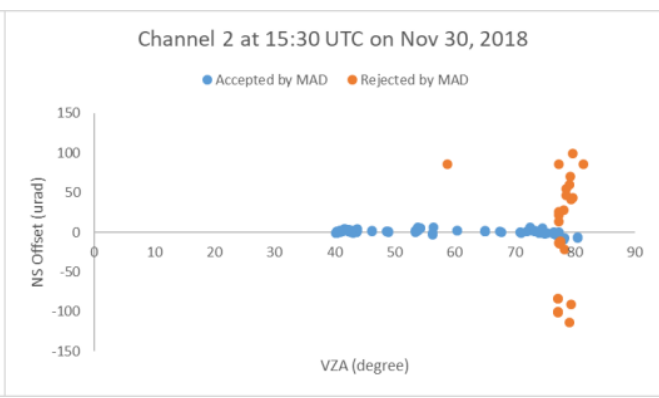

(d)

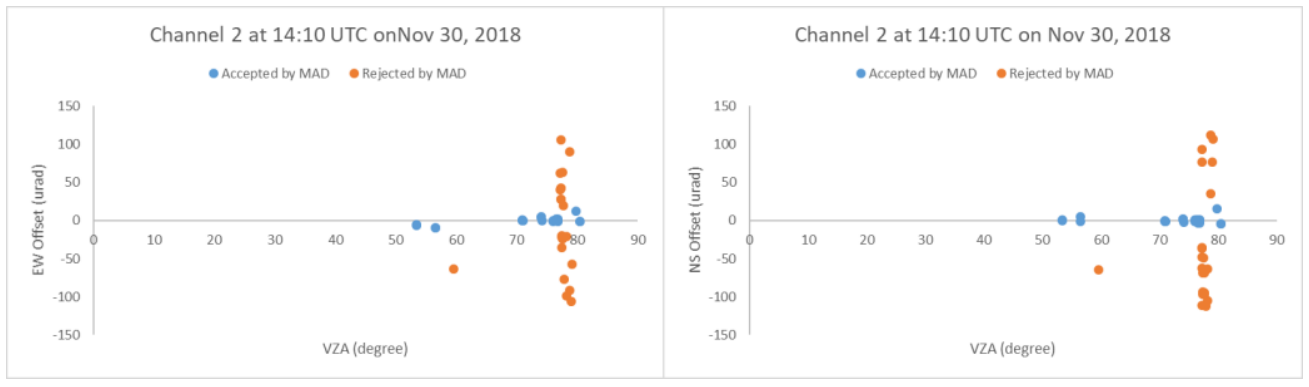

Figure 5. The measured error against VZA at 15:30 UTC (panels a and b) and at 14:10 UTC (panels c and d) on November 30, 2018.

(a)

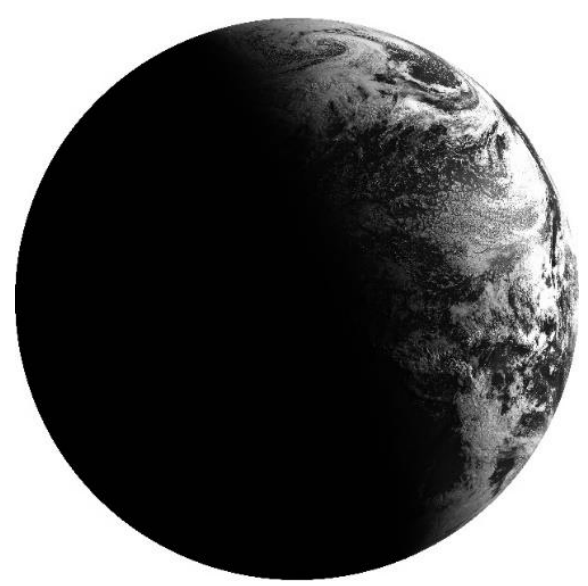

(b)

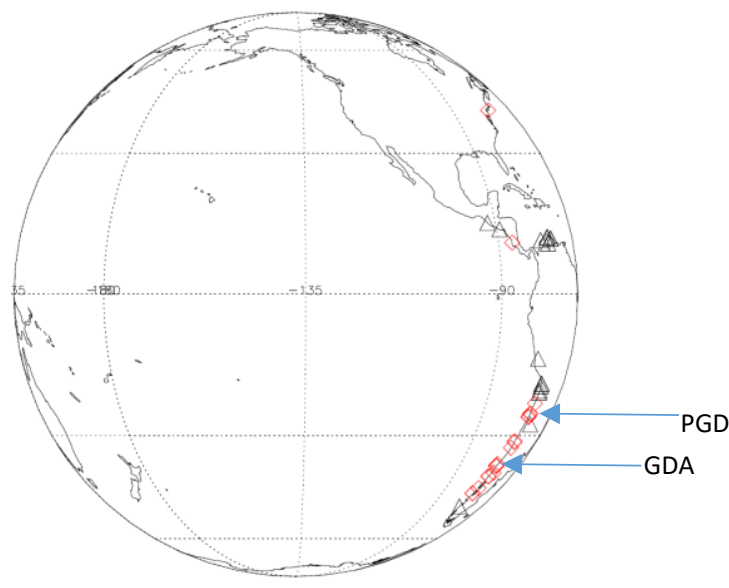

Figure 6. (a) ABI channel 2 Full Disk image at 14:10 UTC November 30, 2018. (b)The valid chips of Channel 2 at 14:10z November 30 2018. The black triangle marks the chips that report the offset passed MAD filter. The red diamond marks the chips were rejected by MAD filter. The location of chip GDA and PGD are marked in (b).

As previous stated, refraction is one possible reason for the low quality measurements. Figure 7 shows the changes of the refraction angle and the linear displacement against VZA. The refraction angle and linear displacement are negligible 
until VZA is 75 degrees, where refraction angle and linear displacement are $0.9 \mu \mathrm{rad}$ and $136 \mathrm{~m}$ respectively. The refraction angle jumps to $1.9 \mu \mathrm{rad}$ and $6.3 \mu \mathrm{rad}$ when VZA equals to 80 and 85 degrees, which corresponds to $448 \mathrm{~m}$ and $2974 \mathrm{~m}$ linear displacement respectively. However, comparing to the variation of measured NAV errors, the effect of refraction is marginal.

(a)

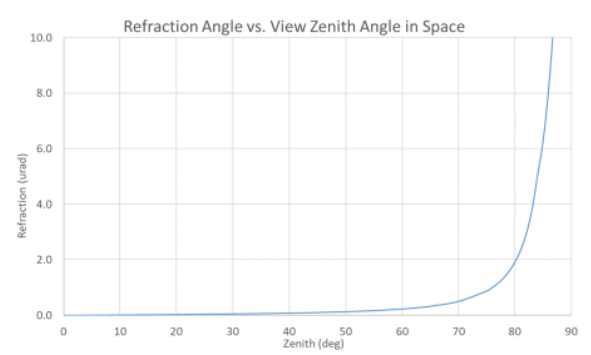

(b)

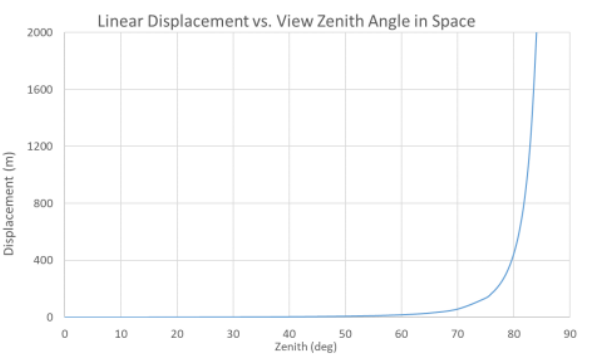

Figure 7. The refraction angle and linear dispatch distance on the ground versus view zenith angle. The plots are retrieved from Table 4 in [11].

The large pixel footprint, when VZA is large, is another possible reason for the low quality measurements. We selected two chip locations, GDA and PGD (Figure 6b), to analyze the reason of the abnormal large measured error when VZA is large. The NAV measurements of these two chips at 14:10 UTC in channel 2 are in Table 2. They reported extremely large measured errors in both directions, which do not align with the results reported by chips with low VZA in the same scene. The low values of analytical measurement uncertainty v2 (aMU2) of these two measurements indicates good quality. aMU2 is a quality indicator of the image registration result [6]. Lower aMU2 value means higher possibility of good quality registration result.

Table 2. The NAV measurements at locations GDA and PGD in channel 2 at 14:10 UTC November 30, 2018

\begin{tabular}{|c|c|c|c|c|c|c|}
\hline Chip Name & EW Error ( $\mu \mathrm{rad})$ & NS Error ( $\mu \mathrm{rad})$ & $\begin{array}{c}\text { Longitude at Chip } \\
\text { Center(degree) }\end{array}$ & $\begin{array}{l}\text { Latitude at Chip } \\
\text { Center (degree) }\end{array}$ & EW MU & NS MU \\
\hline GDA & -22.01 & -97.11 & -73.45 & -37.44 & 1.12 & 1.40 \\
\hline PGD & 27.38 & -110.88 & -70.63 & -25.57 & 1.21 & 2.41 \\
\hline
\end{tabular}

Figure 8 shows the aggregated Landsat chips and corresponding resampled ABI subset at 14:10 UTC. It is obvious that clouds covered most of the ABI subsets at these two chip locations (Figure 8a and 8c). The misleading values of aMU2 are due to two factors: a) the extreme coarse resolution Landsat chips containing much less earth surface information than the chips in the original resolution (Figure 8) and b) the size of chips is very small, only 10 by 10 and 14 by 15 ABI pixels for GDA and PGD respectively. These locations are near the limb of the earth and thus the spatial resolution of the ABI image at these locations are about 8 times coarser than at nadir $(0.5 \mathrm{~km})$. The limited dimension of the subset provides insufficient feature information for the image-matching process and image quality evaluation. The detailed spatial features in the original Landsat chips turn into features in a few pixels after the aggregation, which are difficult to distinguish with the noise in the images. It is not surprising that the IPATS NAV module did not function well with such small aggregated chips. A VZA filter with a threshold at 75 degrees effectively removes such low quality measurements. Figure 9 shows temporal plots for channel 2 per-scene navigation with and without the VZA filter from November 28 to December 1st 2018. The significant standard deviation (STD) of measured NAV errors, about $50 \mu \mathrm{rad}$, in scenes around 14:00 UTC each day dropped to about $2 \mu \mathrm{rad}$ after applying the VZA filter. The drop of the STD indicates the removal of the measurements with excessive errors. 
(a)

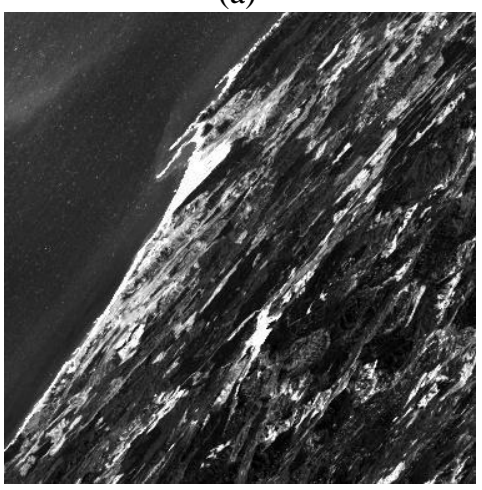

(c)

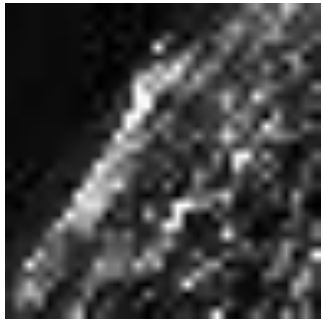

(d)

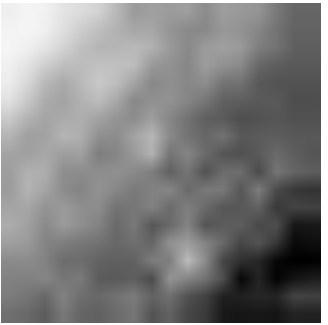

(b)

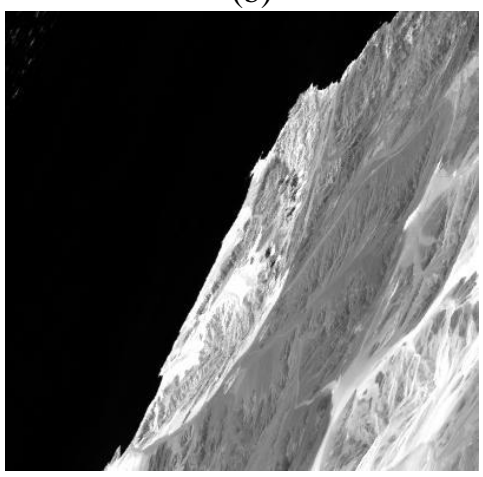

(e)

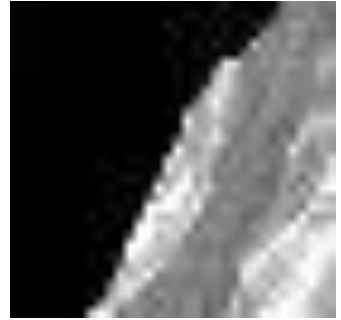

(f)

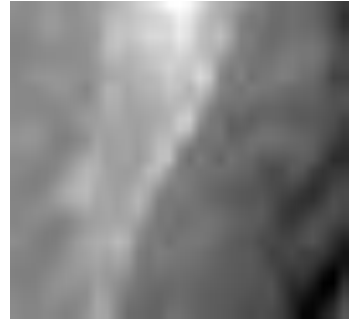

Figure 8. The fine resolution chip images (a) GDA and (b) PGD, and aggregated chip images (c) and (e). Panel (d) and (f) are the ABI subsets corresponding to GDA and PGD at 14:10 UTC November 30, 2018.

(a)

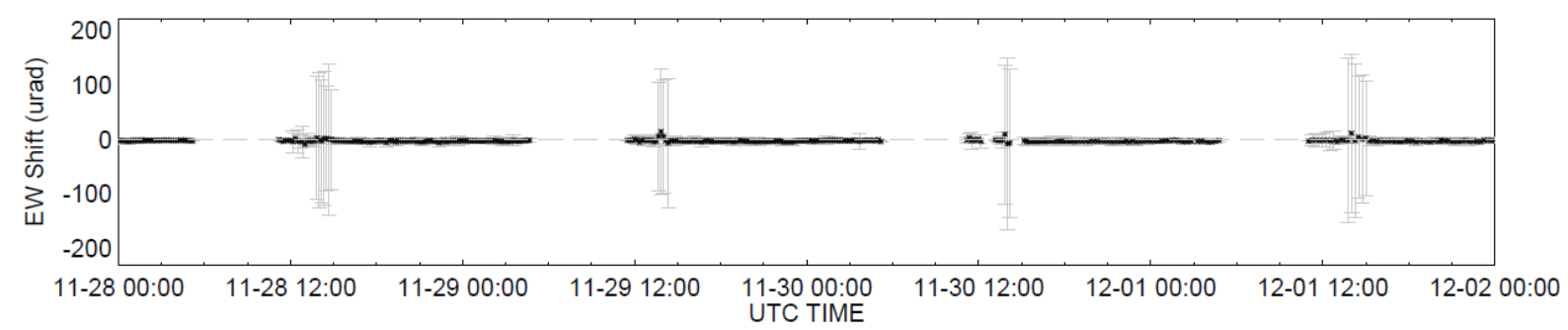

(b)

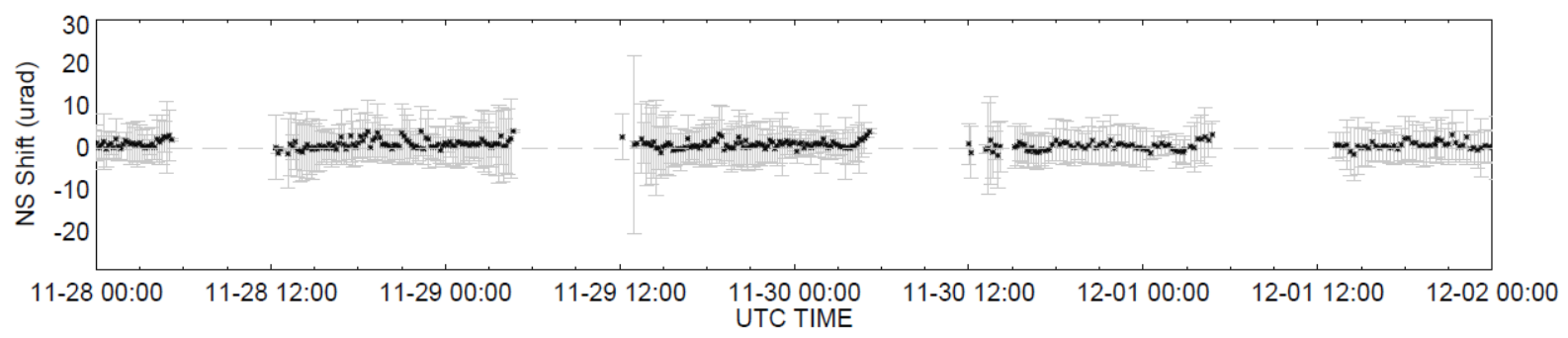

Figure 9. Temporal plot of NAV assessment results at Channel 2 EW direction from November 28 to December 012018. Panel (a) is without VZA filter and panel (b) is with VZA filter applied. The points represent the average NAV measurements in a scene. The error bar is the 3 times STD of the NAV measurements in a scene. 


\section{Long-term ABI NAV Assessment Results}

Figures 10 and 11 show the measured ABI NAV errors of both GOES-16 and GOES-17 ABIs from the start of postlaunch-testing (PLT) to May 19, 2019. Channels 2, 7, and 13 represent VNIR, MWIR, and LWIR focal plane modules, respectively. There are about 2.5 years of GOES-16 record and about 1 year of GOES-17 record. Long-term ABI NAV results capture the on-orbit calibration process of ABI navigation system. In general, GOES-16 NAV error in the NS direction is less than in the EW direction and is more stable over time. GOES-17 NAV errors are comparable in both directions. The gaps in the early stage of PLT are due to the very large NAV errors, which are out of the plot range.
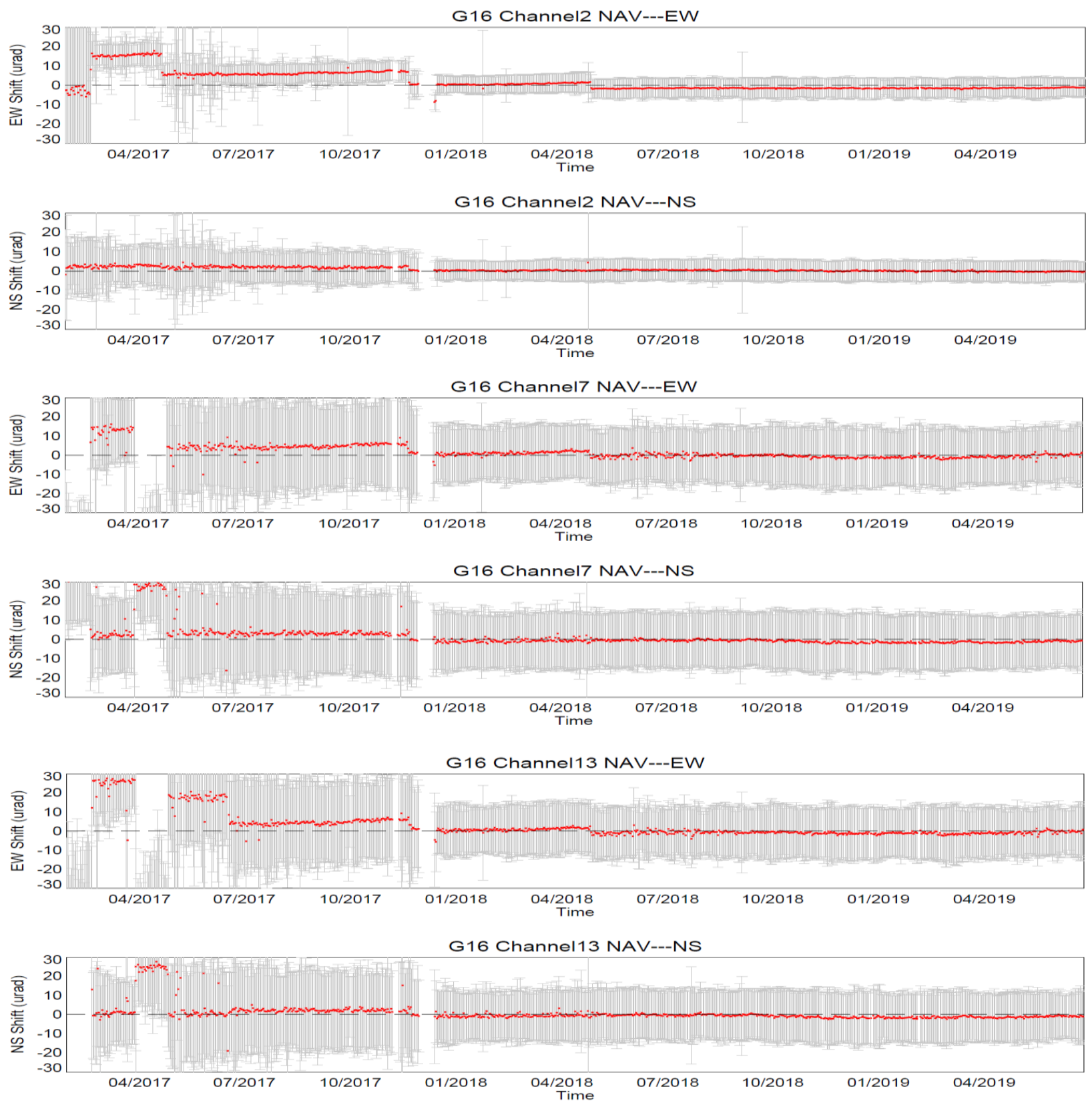

Figure 10. The time series plots of the daily mean and 3 times standard deviation of the GOES-16 ABI NAV errors from the beginning of PLT to June 30, 2019. 

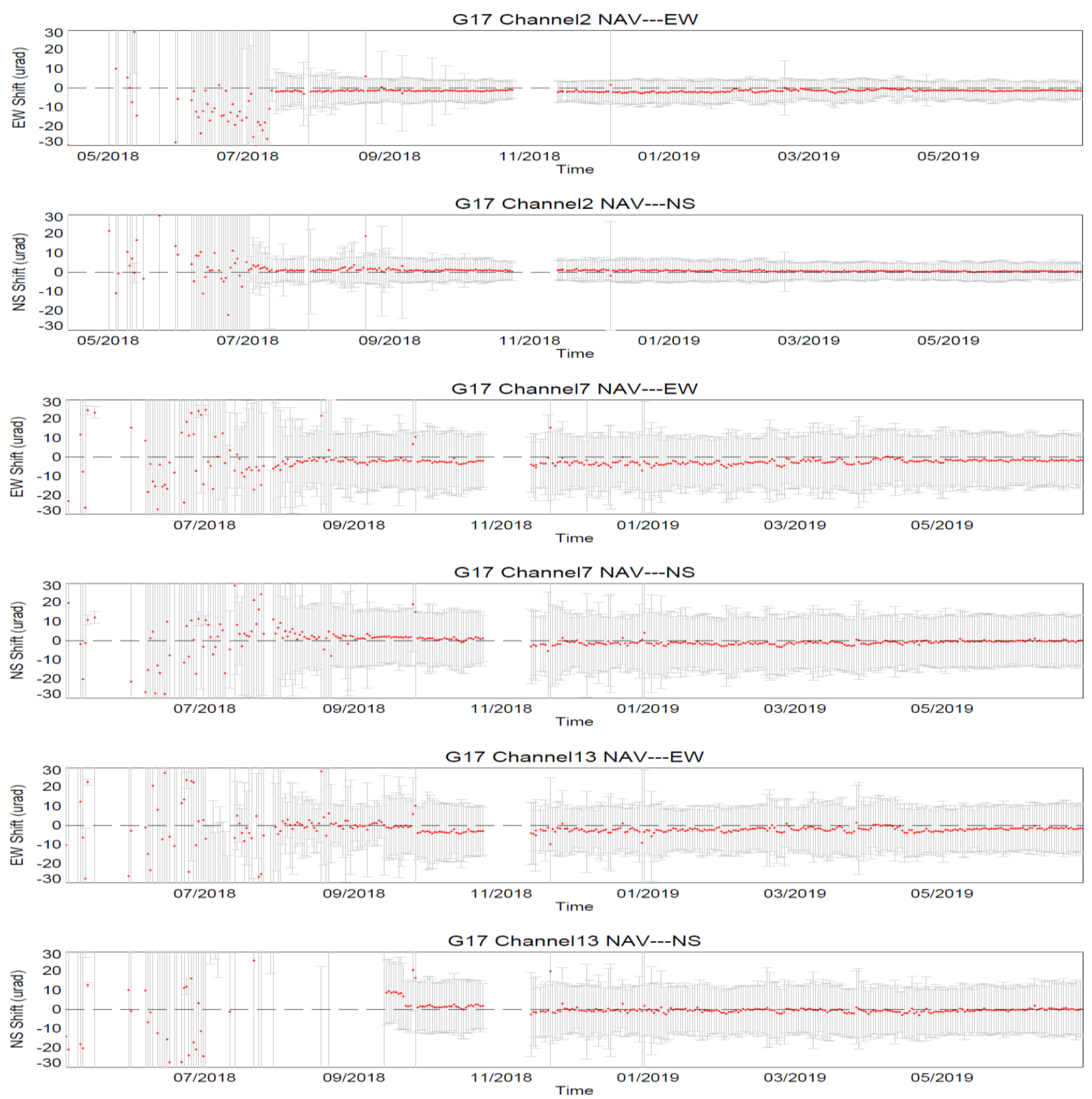

Figure 11. The time series plots of the daily mean and 3 times standard deviation of the GOES-17 ABI NAV errors from the beginning of PLT to June 30, 2019.

The NAV error of GOES-16 in the EW direction of all channels and NS direction for channels 7 and 13 are significant from January 27 to April 27 2017. The time period, when GOES-17 ABI NAV is with significant errors, is from May 1st 2018 to July 11th 2018. Figure 10 shows the NAV long-term trend of GOES-16. The major INR updates in April and November 2017 and April 2018 are obvious in the plots. Two updates in 2017 improved the NAV errors to around $1 \mu$ rad in both directions across all channels. The update in June 2018 removed gradual increase in EW NAV error, which increased about $1 \mu \mathrm{rad}$ in all channels from June 2017 to November 2017 and from November 2017 to April 2018. This is due to a zonal tide term which was missing in the INR calculation. The missing term drifts slowly over time and led to the EW error gradually increasing as observed. Figure 11 is the NAV long-term trend of GOES-17. The NAV errors dropped 
to around $1 \mu \mathrm{rad}$ in VNIR channels and around $2 \mu \mathrm{rad}$ in IR channels after the INR updates in July 2018. There is no significant INR accuracy change observed with other INR updates.

It took 3 months for GOES-17 to bring the NAV accuracy to about 1-2 $\mu$ rad and 11 months for GOES-16 to obtain the similar improvement. The faster improvement of GOES-17 is benefited from the lessons learned from GOES-16 PLT. Currently, the NAV errors of GOES-17 is about $1 \mu \mathrm{rad}$ larger than GOES-16.

\section{Conclusions}

IPATS has been implemented to continuously monitor ABI INR performance since the start of GOES-16 PLT. The generated INR metrics have been used to help tuning of both ABI navigation systems to achieve excellent image navigation and registration accuracy requirements. This study shows that the IPATS measurement accuracy is sufficient to the accuracy requirement of the INR assessment. IPATS is not a static system. Additional filters and/or sub-procedures were developed when the demand emerged, e.g. the development of STAND and VZA filters in PLT of GOES-16 and GOES17 respectively. NAV INR accuracy improved with updates and tuning in PLT. Currently, NAV errors are about 1-2 $\mu$ rad for all assessed channels of both ABIs.

\section{ACKNOWLEDGEMENT}

The IPATS activity is supported by the NASA/NOAA GOES-R Series Project. The authors acknowledge contributions by the many members of the GOES-R Series Flight Project (including the spacecraft and instrument designers and builders), the GOES-R Series Ground Project and the NOAA GOES-R Series Calibration Working Group, whose efforts made the noted levels of ABI INR performance possible.

\section{REFERENCE}

[1] Miller, S. D., Schmidt, C. C., Schmit, T. J. and Hillger, D. W. “ A case for natural colour imagery from geostationary satellites, and an approximation for the GOES-R ABI", International Journal of Remote Sensing, 33:13, 3999-4028, DOI: $10.1080 / 01431161.2011 .637529$ (2012).

[2] Chapel, J., Stancliffe, D., Bevacqua, T., Winkler, S., Clapp, B., Rood, T., Gaylor, D., Freesland, D. and Krimchansky, A. "Guidance, Navigation, and Control Performance for the GOES-R Spacecraft", CEAS Space Journal, DOI 10.1007/s12567-015-0077-1 (2015).

[3] Schmit, T. J., Griffith, P., Gunshor, M. M., Daniels, J. M., Goodman, S. J. and Lebair, W. J. "A closer look at the ABI on the goes-r series". Bulletin of the American Meteorological Society, 98(4), 681-698. DOI 10.1175/BAMS-D-1500230.1 (2017).

[4] 'Product Definition and Users' Guide (PUG) Volume 3: Level 1B Products for Geostationary Operational Environmental Satellite R Series (GOES-R) Core Ground Segment, Revision D”, http://www.goesr.gov/users/docs/PUG-L1b-vol3.pdf (May 2015).

[5] De Luccia, F. J., Houchin, S., Porter, B. C., Graybill, J., Haas, E., Johnson, P. D., Isaacson, P. J., Reth, A. D. "Image navigation and registration performance assessment tool set for the GOES-R Advanced Baseline Imager and Geostationary Lightning Mapper", Proc. SPIE 9881, Earth Observing Missions and Sensors: Development, Implementation, and Characterization IV, 988119 (2016).

[6] Tan, B., Dellomo, J., Wolfe, R. E., Reth, A. D., "GOES-16 ABI navigation assessment," Proc. SPIE 10764, Earth Observing Systems XXIII, 107640G (2018).

[7] Grycewicz, T. J., Tan, B., Isaacson, P. J., De Luccia, F. J., Dellomo, J., "Avoiding stair-step artifacts in image registration for GOES-R navigation and registration assessment," Proc. SPIE 9972, Earth Observing Systems XXI, 99720T (2016).

[8] Schaaf, C. B., Gao, F., Strahler, A. H., Lucht, W., Li, X., Tsang, T., Strugnell, N. C., Zhang, X., Jin, Y., Muller, J., Lewis, P., Barnsley, M., Hobson, P., Disney, M., Roberts, G., Dunderdale, M., Doll, C., P d'Entremont, R., Hu, B., Liang, S., Privette, J. L., Roy, D., "First operational BRDF, albedo nadir reflectance products from MODIS". Remote Sens. Environ., 83, pp. 135-148 (1999). 
[9] Xin, Q., Woodcock, C. E., Liu, J., Tan, B., Melloh, R. A., Davis, R. E., "View angle effects on MODIS snow mapping in forests", Remote Sens. Environ., 118, pp. 50-59 (2012).

[10] Wan, Z., Zhang, Y., Zhang, Q., Li, Z. L.,"Quality assessment and validation of the MODIS global land surface temperature", International Journal of Remote Sensing, 25:1, pp. 261-274 (2004).

[11] Noerdlinger, P. D., "Atmospheric refraction effects in Earth remote sensing”, ISPRS Journal of Photogrammetry \& Remote Sensing vol 54, pp. 360-373 (1998). 